\title{
Pancreatic surgery during COVID-19 pandemic: major activity disruption of a third-level referral center during 2020
}

\author{
Giovanni Marchegiani ${ }^{1}$ (i) $\cdot$ Giampaolo Perri $^{1} \cdot$ Beatrice Bianchi ${ }^{1} \cdot$ Alessandro Esposito $^{1} \cdot$ Luca Landoni $^{1}$. \\ Luca Casetti $^{1}$ - Massimiliano Tuveri ${ }^{1}$. Giuseppe Malleo ${ }^{1}$. Salvatore Paiella ${ }^{1}$ - Martina Fontana ${ }^{1}$ - Antonio Pea ${ }^{1}$. \\ Matteo De Pastena ${ }^{1} \cdot$ Roberto salvia ${ }^{1} \cdot$ Claudio Bassi $^{1}$
}

Received: 7 September 2021 / Accepted: 18 October 2021 / Published online: 23 October 2021

(C) Italian Society of Surgery (SIC) 2021

\begin{abstract}
Introduction The COVID-19 pandemic has severely limited the access to cancer surgery, but it is not known to what extent referral centers for pancreatic diseases were affected by its outbreak. The aim of this study is to describe the effect of COVID19 pandemic on a third-level referral center for pancreatic surgery in Italy.

Methods The 2020 activity of The Pancreas Institute of the University of Verona was reviewed, comparing different phases of the COVID-19 pandemic outbreaks using the pre-COVID era as a control. Endpoints were the overall caseload of pancreatic resections, surgical waiting list, administration of preoperative therapy, major morbidity and mortality, residents' training; number of inpatients beds, outpatient visits/procedures/diagnostics.

Results In 2020, there was an overall significant reduction of pancreatic resections performed (394 vs. 506 in 2019), particularly during the first (March-May) and second (October-December) COVID-19 outbreaks, with an all-time-low of 16 resections/months in April (compared to 43 average resection/month in 2019). The rates of major morbidity (Clavien-Dindo $\geq 3$ ) and mortality were similar to 2019 ( 16 vs $12 \%, p=0.11$ and 3 vs $2 \%, p=0.29$, respectively). During the first and second outbreaks resident's training, inpatient beds, outpatient visits, diagnostics, and procedures were severely impaired, while the waiting list for up-front cancer resections and the use of preoperative chemotherapy concomitantly raised.

Conclusion The COVID-19 pandemic has severely disrupted the activity of a third-level referral center for pancreatic surgery, affecting the access to cancer surgical procedures and raising concerns regarding the solidity of the current centralization model.
\end{abstract}

Keywords COVID-19 $\cdot$ Pancreatic surgery $\cdot$ Pandemic $\cdot$ Waiting list $\cdot$ Pancreatic cancer

\section{Introduction}

Since March 2020, the worldwide healthcare system faced the explosive outbreak of the COVID-19 pandemic [1]. Italy was one of the first and most heavily hit countries [2]. To date, a total of 4.275 .846 cases and 127.831 total deaths are reported in the country since the beginning of the pandemic [3]. During the first "wave" of new cases and deaths, the capacity of both hospitals and intensive care units (ICUs) was critically limited, with a deep impact on surgical care

Giovanni Marchegiani

giovanni.marchegiani@aovr.veneto.it

1 Department of General and Pancreatic Surgery-The Pancreas Institute, Verona University Hospital Trust, Piazzale Scuro 10, 37134 Verona, Italy and a heavy restriction of elective surgery in favor of emergency surgery $[4,5]$. After an all-time low of new cases reached during the summer, a second outbreak hit the country in the fall with unprecedented peaks of cases $(41.188$, November 13th 2020) and deaths (992, December 3rd 2020) [6]. Oncologic patients were treated according to clinical priority and different resources available in each territorial referral centers.

Pancreatic cancer surgery often requires ICU admission, longer times of hospitalization, and in general a demanding deployment of resources from the healthcare system. For these reasons, despite the presumed importance of a timely treatment due to the high biological aggressiveness of their disease, patients affected by pancreatic cancer have probably been even more affected by COVID-19 related restrictions and delays, as they represent a difficult "target" to prioritize 
during the pandemic [7-10]. It has been already extensively demonstrated that higher hospital volume is strongly associated with lower morbidity and mortality after pancreatic surgery. [11-13] As a result, during the past decades, there was a growing trend toward referral of patients to high-volume hospitals, worldwide as well as in Italy [14]. However, it is not known to what extent referral centers for pancreatic diseases were affected by the COVID-19 outbreak, and if (and how) they were able to react and adapt while coexisting with the pandemic restrictions.

The Pancreas Institute of Verona University Hospital represents the leading Italian tertiary referral center for pancreatic cancer surgery. The aim of this study is to describe the effects of COVID-19 pandemic on a high-volume center for pancreatic surgery in Italy and to assess whether this model was able to withstand the pressure of the pandemic. The entire center's activity throughout the year 2020 was reviewed according to the different phases of COVID-19 outbreaks, and compared to 2019 as the pre-COVID era, to evaluate the fluctuations of volume and quality of assistance during the pandemic.

\section{Methods}

This study was approved by the local ethics committee (Comitato Etico delle Province di Verona e Rovigo, approval number 1101CESC). The activity of the Verona Pancreas Institute during the year 2020 was reviewed starting from January 1st until December 31st and displayed on a monthly basis. The collected data included: number of pancreatic resections (any kind); number of pancreatic resections performed after the administration of preoperative therapy; number of pancreatic resections performed by residents as first surgeons; major morbidity (defined as Clavien-Dindo greater or equal to 3) [15]; 90 day in-hospital mortality; number of inpatients hospital beds occupied daily in the surgical department; number of specialistic outpatient visits; number of outpatients procedures (endoscopic ultrasound [EUS]; endoscopic retrograde cholangiopancreatography [ERCP]; esophagogastroduodenoscopy [EGDS]; percutaneous fine needle aspirations [FNA]); number of outpatients radiological examinations; waiting list for malignant pancreatic/periampullary pathologies. The waiting list was defined starting from the date of scheduling for surgery after the first evaluation for suspected pancreatic malignancy by the surgeon (including possible time needed for cytological diagnosis/biliary stenting) to the date of surgery. Only patients with a final histopathologic diagnosis of malignancy (i.e., pancreatic adenocarcinoma; adenocarcinoma of the Vater's ampulla/duodenum; cholangiocarcinoma; invasive cancer arising from intraductal papillary mucinous neoplasm) and who were resected up-front (without administration of preoperative therapy) were included in the waiting list calculation. To correlate the fluctuation of the clinical activity with the ongoing pandemic, epidemiologic data depicting the COVID-19 pandemic in Italy were retrieved from https://www.worldometers.info/coronavirus and graphically expressed as highest peaks of daily new cases and deaths reached during each month.

The Italian healthcare system, of which the Verona Hospital is part, is currently organized on a public, regional hub-and-spoke model. This system arranges service delivery into a network consisting of an anchor establishment (hub) which offers a full array of services and is represented by the referral center, complemented by secondary establishments (spokes) which offer more limited services.

\section{Statistical analysis}

Continuous variables are expressed as the means and (SD) values or as median values with ranges and were compared using the independent samples $t$ test or the Mann-Whitney test, as appropriate. Categorical variables are expressed as frequencies with percentages and were compared using the Chi-square test or Fisher's exact test in case of small expected frequencies. All the tests were two-tailed. $P<0.05$ was considered statistically significant. Statistical analyses were performed using STATA14 for Windows.

\section{Results}

\section{The COVID-19 outbreaks in Italy during 2020}

The trends of COVID-19 daily new cases and deaths in Italy during 2020 are reported in Fig. 1. The highest peaks of daily new cases and deaths were reached during the months of March, April, and May (1st outbreak), and October, November, and December (2nd outbreak).

\section{Institutional surgical activity}

Supplementary Table 1 displays the Institutional surgical activity during 2020. Overall, 394 pancreatic resections were performed during 2020, compared to 506 in 2019 (Fig. 2). The number of monthly pancreatic resection dropped during first pandemic outbreak reaching an all-time-low in April $(n=16)$. The number of resections increased again during summer, with a peak in October $(n=46)$ and dropping down again in December $(n=24)$ during the second outbreak. Of note, an average of 42 pancreatic resections per month were performed in 2019. 


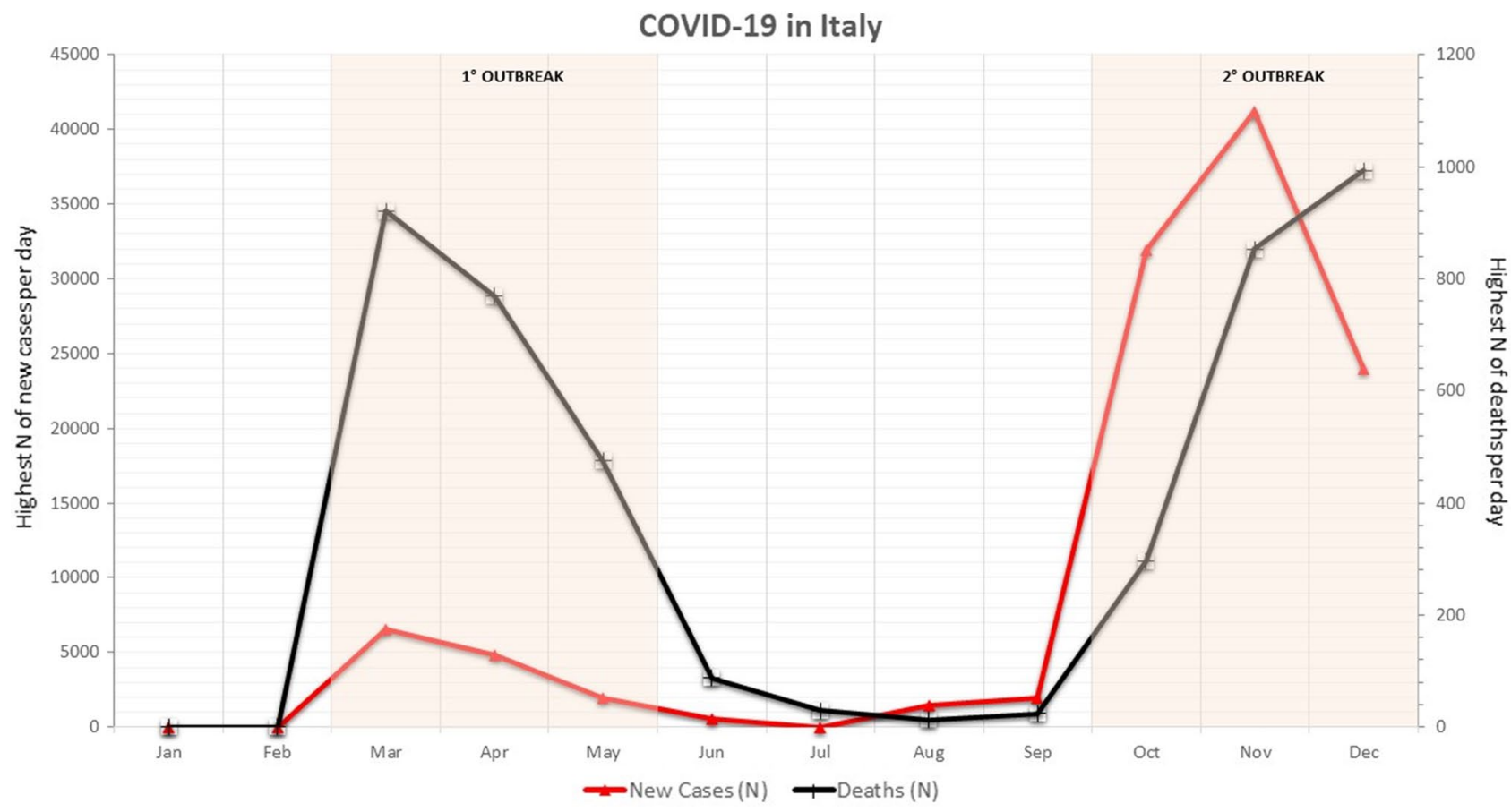

Fig. 1 Highest peaks of COVID-19-related daily new cases (red line) and deaths (black line) for each month of 2020 in Italy

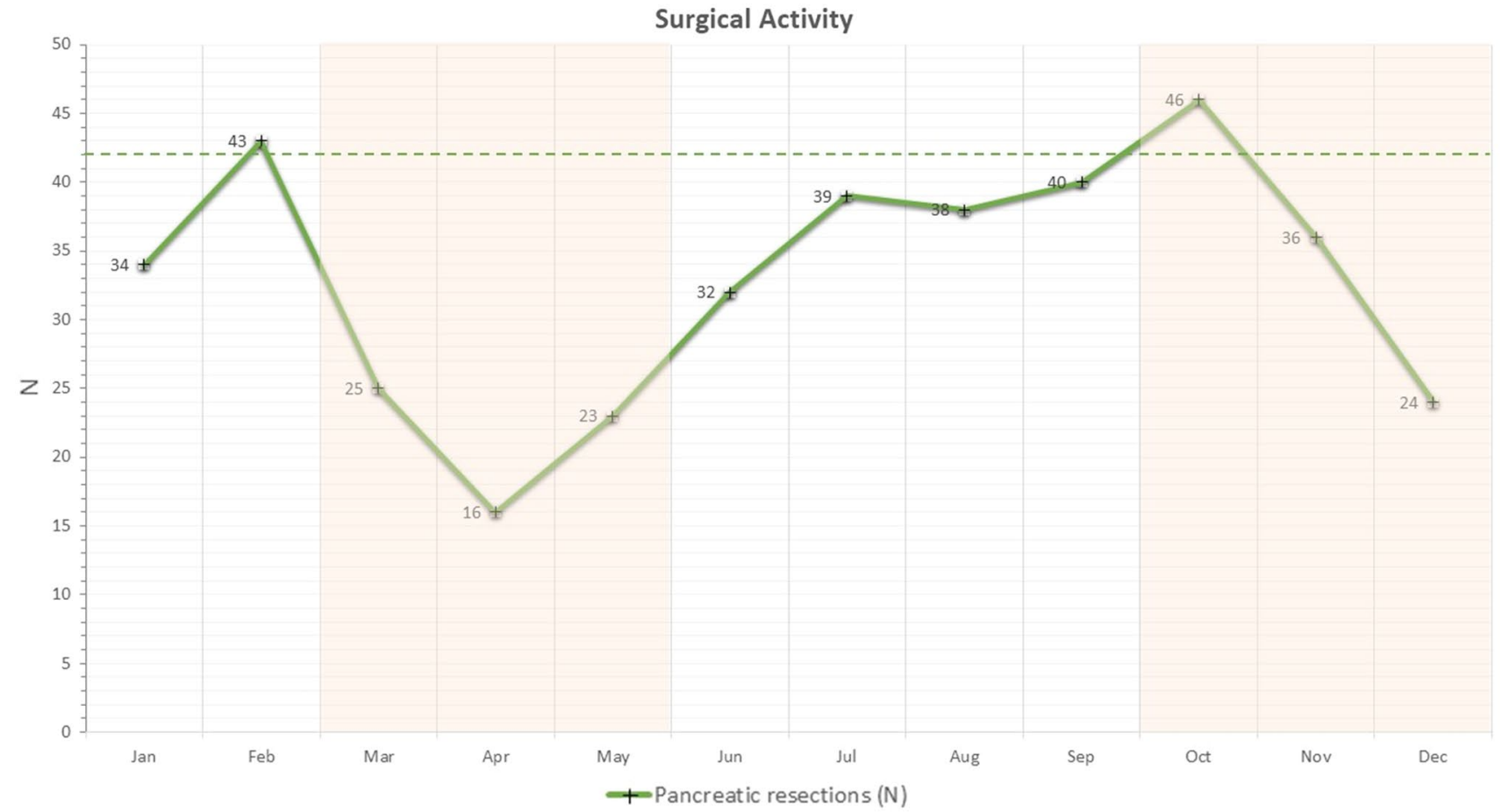

Fig. 2 Number of pancreatic resections (green line) for each month of 2020 (dotted green line: monthly average of 2019)

\section{Morbidity and mortality}

Compared to 2019, no significant differences were found during 2020 in terms of surgical major morbidity (16 vs $12 \% ; P=0.11$ ) and mortality ( 3 vs $2 \% ; P=0.29$ ). In Fig. 3, major morbidity and mortality during each month are reported. They increased during the first COVID-19 outbreak, peaking in March with a 36\% rate of major 


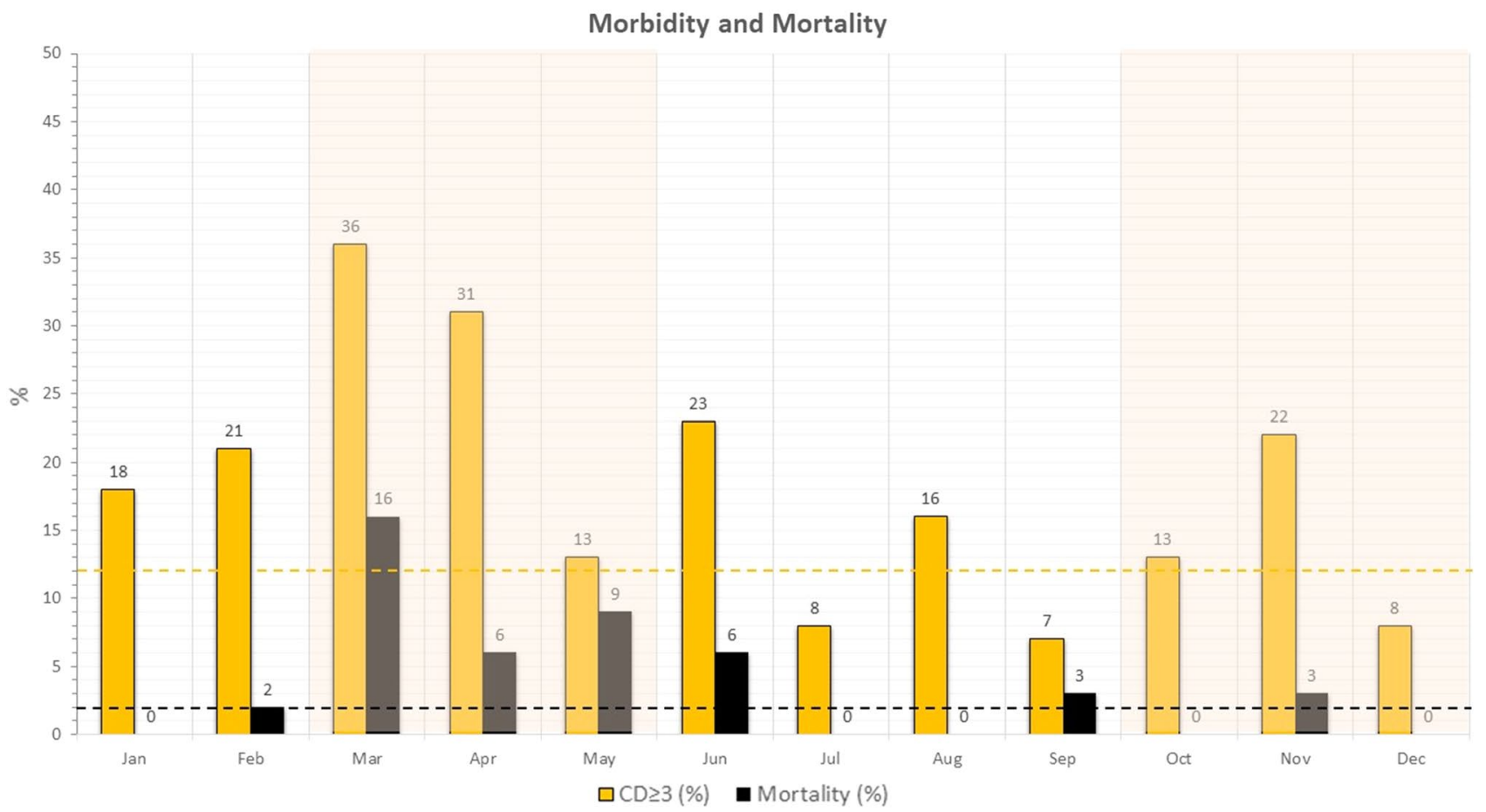

Fig. 3 Surgical major morbidity (yellow bars) and mortality (black bars) for each month of 2020 (dotted yellow and black lines: average major morbidity and mortality during 2019)

morbidity and $16 \%$ of in-hospital mortality. During the second half of 2020, including the months of the second outbreak, major morbidity and mortality remained similar or lower to that of 2019.

Overall, 13 patients (3\%) died during 2020. Among them, 4 suddenly died within the first 10 post-operative days without surgical complications (2 in March, 1 in May, and 1 in December).

\section{Waiting list and preoperative therapy}

The days of waiting lists for up-front cancer resections and the rate of resections after preoperative therapy in 2020 are showed in Fig. 4. The median waiting list in 2020 was 57 days, compared to 45 days in 2019 ( $P=0.003,95 \%$ CI 19-4). During the first outbreak, the waiting list increased peaking up to 85 and 88 days in March and May, respectively, and then decreased again during the summer. The rate of resections performed after preoperative therapy increased contextually, reaching $61 \%$ in June, and in general remained of around $50 \%$ for most of the year. In total, 165 (42\%) patients were resected after preoperative therapy in 2020, compared to $177(35 \%)$ in $2019(P=0.04)$.

\section{Training}

The rate of pancreatic resections performed by senior residents as first surgeons in 2020 is showed in Fig. 5. In $2020,16 \%(n=64)$ of total resections were performed by residents, compared to $17 \%(n=86)$ in $2019(P=0.8)$. Resections performed by residents dropped down to null in April during the first outbreak and remained below the 2019 average (dotted line) for the majority of 2020.

\section{Outpatient clinics and inpatient hospital beds}

Figure 6 displays the median number of inpatient hospital beds occupied daily and the total number of pancreatic outpatient clinics for each month of 2020. A monthly median of 33 beds/day and 600 visits were reported in 2019, compared to 29 beds/day ( $P=0.02,95 \%$ CI 5-7) and 488 visits $(P=0.07,95 \%$ CI 11-235) in 2020. Both decreased during the first COVID-19 outbreak (down to 14 beds/day and 88 visits in April), then increased again in the summer, and went back down during the second outbreak. 


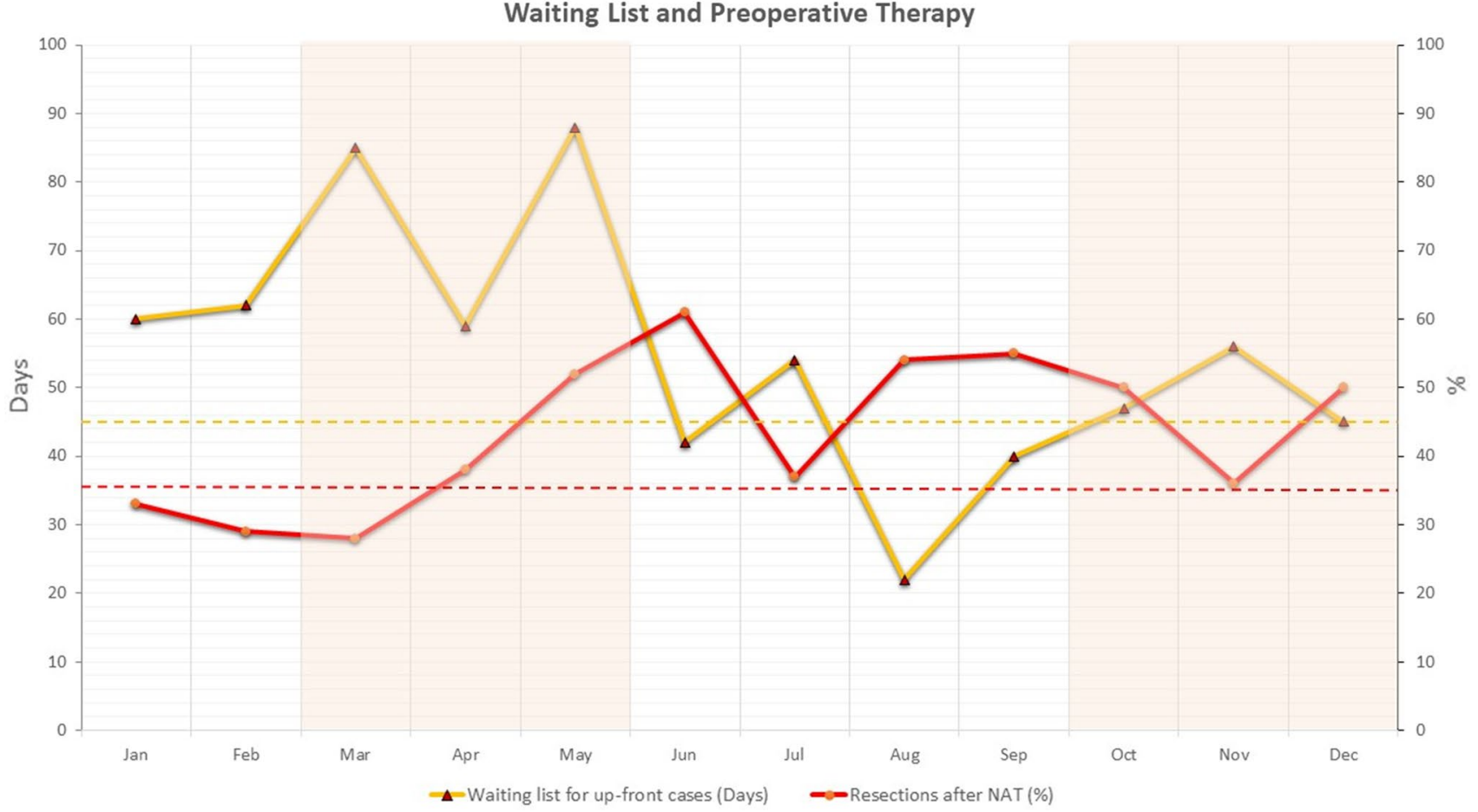

Fig. 4 Days of waiting list for up-front resections (yellow line) and percentage of resections performed after preoperative therapy (red line) for each month of 2020 (dotted yellow and red lines: average waiting list and rate of preoperative therapy administration during 2019)

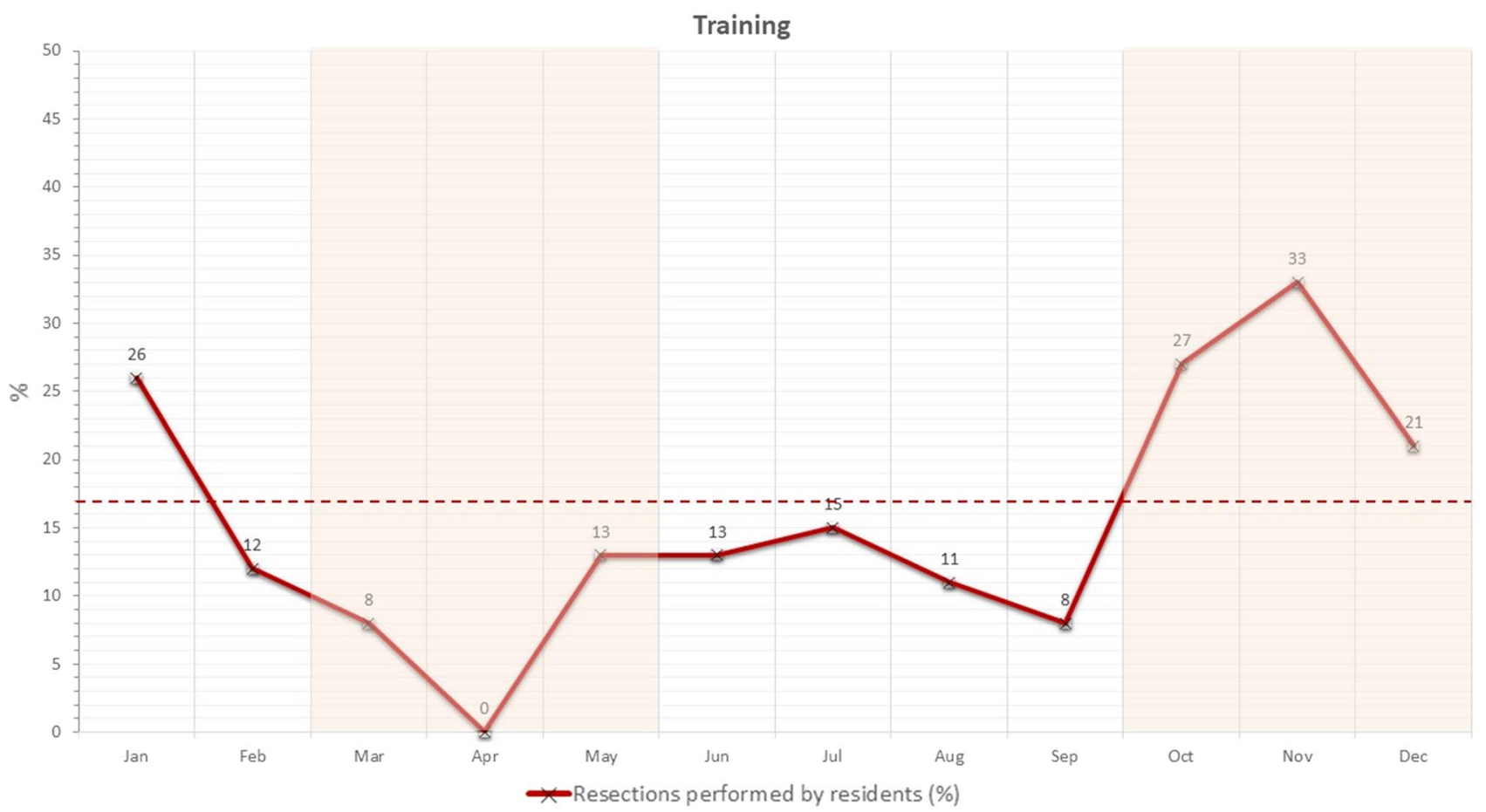

Fig. 5 Rates of pancreatic resections performed by senior residents as first surgeons (red line) for each month of 2020 (dotted red line: average rate of resections performed by residents during 2019) 


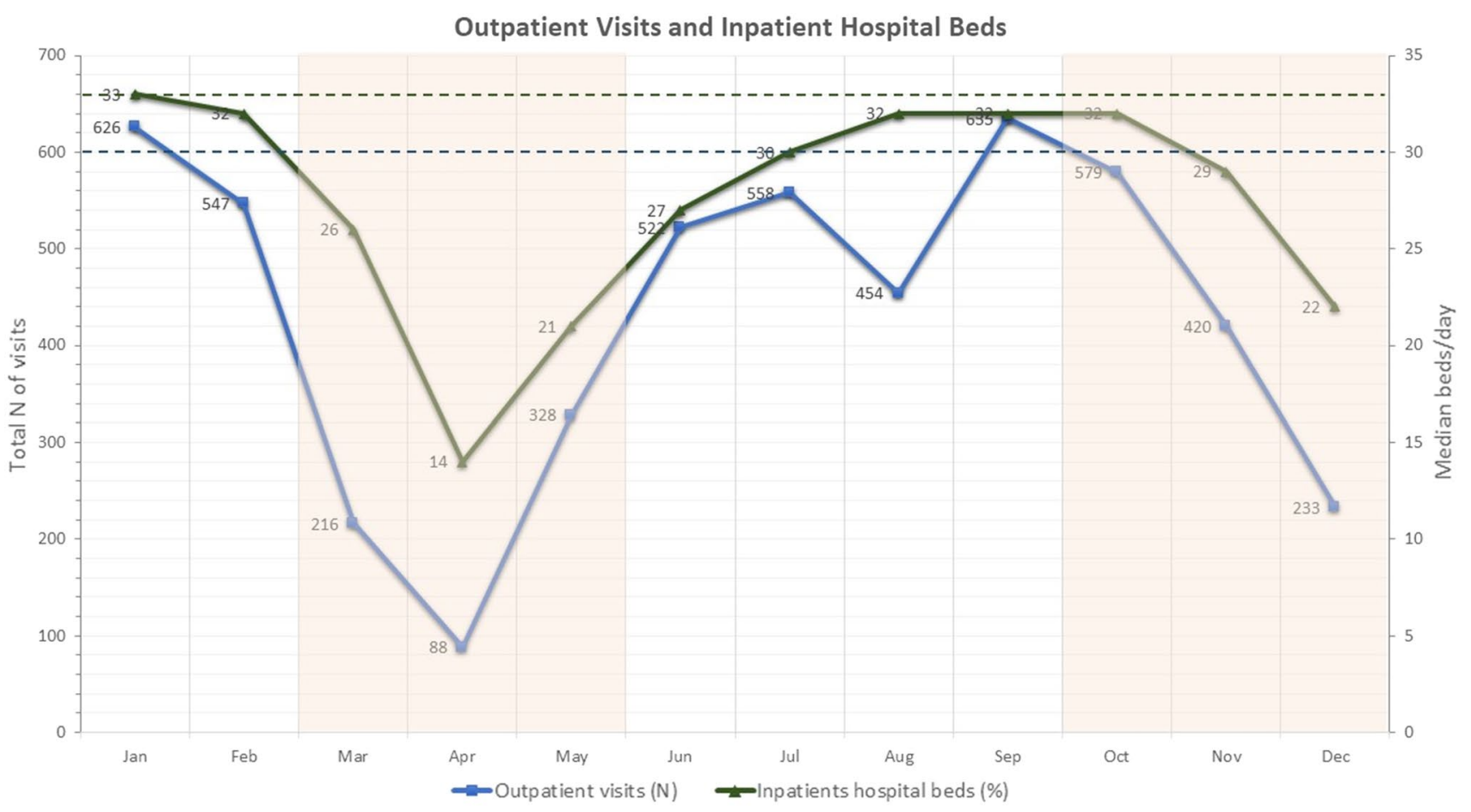

Fig. 6 Median of inpatient hospital beds occupied daily (green line) and total number of specialistic outpatient visits (blue line) for each month of 2020 (dotted green and blue line: monthly average number of beds occupied daily and total number of visits during 2019)

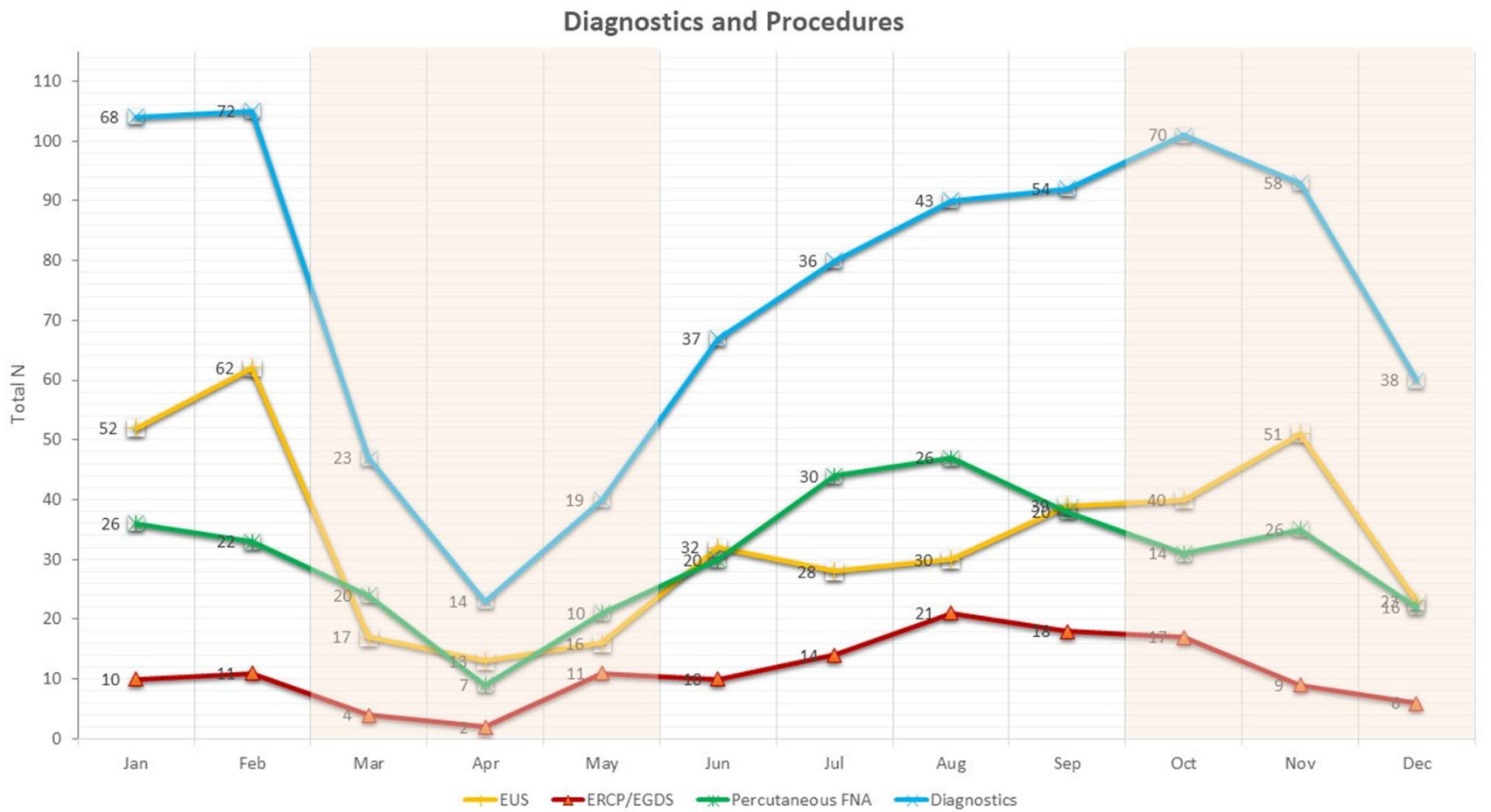

Fig. 7 Number of EUS (yellow line), ERCP/EGDS (red line), percutaneous FNA (green line), and radiographic diagnostics (blue line) performed during each month of 2020 


\section{Diagnostics and procedures}

Total numbers of EUS, ERCP/EGDS, percutaneous FNA, and radiographic diagnostics performed during each month of 2020 are showed in Fig. 7. The number of all diagnostics and procedures performed dropped down during both the first and second outbreaks of COVID-19 pandemic.

\section{Discussion}

The COVID-19 pandemic disrupted the activity of an Italian third-level referral center for pancreatic surgery. In 2020, especially during the first (March-May) and second (October-December) outbreaks, the number of surgical resections, inpatient beds, outpatient clinics, diagnostics, and procedures were impaired. Concomitantly, the waiting list for up-front cancer resections and the rate of preoperative therapy administration dramatically raised.

The timely access to surgery for patients with cancer is considered a quality-of-care indicator. The outbreaks and spread of COVID-19 determined a global reduction of routine hospital services, decreasing the access to complex cancer surgery worldwide $[4,5,16-18]$. In Italy, the oncological hub-and-spoke program did not seem to work adequately either, as surgical oncology suffered a remarkable reduction of its activity, resulting in a doubled waiting list [19]. This unexpected contingency raised several concerns about the widespread tendency to address patients requiring major cancer surgery to few high-volume hospitals. Despite a detrimental effect of the waiting list elongation on survival has never been clearly demonstrated for pancreatic cancer, it is well known that the clinical progression from low-stage to advanced-stage disease is rapid in this disease. Moreover, reducing the time to surgery seems to affect the increase in cancer size and the rates of radical resections [20-22].

The institutional caseload was reduced by more than $20 \%$ during 2020. This finding is in line with most centers performing pancreatic surgery. In a recent survey, $62 \%$ of the responders from international referral centers declared to have performed less pancreatic surgery because of the COVID-19 pandemic, reducing their median weekly pancreatic resection rate from 3 to 1 [8].

The reduction of the surgical activity was not steady or gradual, but rather sudden and severe, mirroring the waves of COVID-19 new cases in the country. During the first outbreak, to avoid a complete shutdown of the surgical activity, the operating theaters and part of the inpatient beds were moved to the twin University hospital, where the ICU capacity was less limited. The surgical team was provided from the same Pancreas Unit, operating back and forth between the two hospitals.
Despite the overall rates of major morbidity and mortality in 2020 were comparable to that of 2019 , and with all the limitations of dealing with small numbers, it is difficult not to correlate the striking increase in major morbidity and mortality of March, April, and May with the disorganization resulted from the sudden jamming of the healthcare machine. Notably, all patients undergoing surgery were tested with molecular nasal and oropharyngeal swabs, prior to admission and again during hospitalization in case of symptoms. Only one patient tested positive after surgery, due to mild respiratory symptoms. Therefore, it is not possible to ascribe the worse surgical outcomes to a concomitant COVID-19 infection. However, all the early perioperative sudden deaths of 2020 (four patients) happened within the COVID-19 waves.

As a result of the reduction in the surgical activity, the waiting list raised considerably. To maintain disease control and as a bridge to surgical resection, chemotherapy has been proposed as an alternative for patients also with resectable pancreatic cancers awaiting surgery delayed due to COVID-19 [23, 24]. In June, the waiting list for up-front surgery went back down to 42 days, but around $60 \%$ of pancreatic resections for all pathologies were carried out after the administration preoperative chemotherapy (a rate almost doubled compared to 2019). Despite such strategy is currently accepted by many Cancer Centers worldwide, therapeutic decisions in terms of treatment sequencing and time-windows to undergo surgery after preoperative therapy were frequently not evidence-based in this scenario. While it is still too early to evaluate the actual impact of these policies on survival, their possible detrimental effects are to be registered in the long and yet elusive list of COVID-19 collateral damages. Moreover, surgery for benign or premalignant lesions such as intraductal papillary mucinous neoplasms (IPMNs), serous (CAS) and mucinous (CAM) cystadenomas, and G1 neuroendocrine tumors (NETs) was completely suspended, with unknown effects on the possible disease progression of tumors in which prophylactic resection would have been otherwise indicated.

To shorten the waiting list during the summer, given the limited available resources, multiple pancreatectomies (including pancreatoduodenectomies) were often scheduled in a single daily operating session. In this scenario, experienced surgeons are forced to work with maximum effort and at the limit of their own abilities to fully exploit the available operating sessions. Therefore, there is reduced room for training, as the fundamental prerequisites for the transmission of practical knowledge in a safe, profitable way are missing [25].

Less obvious, but equally important, are the collateral damages due to the hundreds of outpatient clinics, diagnostic, and procedures that were canceled or never scheduled to avoid possible risk of contagion for patients. The 
implementation of telemedicine could only partially mitigate the inconveniences related to canceled endoscopic and percutaneous procedures which were reserved for patients with malignancies.

This study provides an opportunity to reflect on the role of high-volume centers for pancreatic surgery in Italy, and its potential reorganization since this model failed under the pressure of the pandemic Coronavirus disease. It became evident that, despite the hard lesson learned during the first outbreak, to allocate the resources necessary to avoid a recrudescence of the activity disruption during the second outbreak were either not possible, or not a priority [10]. At the time of writing, despite the vaccination campaign, the activity of this referral center still varies with the tidal of the regional COVID-19 contagions.

A new strategy to overcome the failure of centralization standard could be the reinvention of the current hub-andspoke organization model. The hub hospitals should probably be declared as "Covid-free", directing all patients found positive at nasopharyngeal test for SARS-Cov2 to peripheral hospitals where elective surgery for non-oncological pathologies could be postponed. The hubs should be able to meet certain requirements such as the possibility of hospitalization and treatment thanks to the presence of specific operating rooms and a "Covid-free" post-operative ICU. An organization of this kind could possibly face the pandemic in a most effective way by allowing the high-volume hospital to maintain its standards of care.

A main limitation of the present study is its monocentric nature. Future multicentric, collaborative studies will hopefully provide a wider and more precise picture of the situation of pancreatic surgery in Italy during the pandemic, further exploring the efficacy of the centralization model.

In conclusion, this study describes the major activity disruption of a high-volume center for pancreatic surgery in Italy due to the COVID-19 outbreak of 2020. The access to cancer care was severely impaired, raising concerns regarding the solidity of the current centralization model.

Supplementary Information The online version contains supplementary material available at https://doi.org/10.1007/s13304-021-01197-y.

Funding This paper was not funded.

\section{Declarations}

Conflict of interest The authors declare that they have no conflicts of interest to declare.

Ethical approval This retrospective chart review study involving human participants was in accordance with the ethical standards of the institutional and national research committee and with the 1964 Helsinki Declaration and its later amendments or comparable ethical standards. The Human Investigation Committee of the University of Verona approved this study with the number: 298CESC.
Informed consent Informed consent was obtained from all individual participants included in the study.

\section{References}

1. Mahase E (2020) China coronavirus: WHO declares international emergency as death toll exceeds 200. BMJ 368:m408

2. Remuzzi A, Remuzzi G (2020) COVID-19 and Italy: what next? Lancet Lond Engl 395:1225-1228

3. WHO Coronavirus (COVID-19) Dashboard Available from: https://covid19.who.int. Accessed 16 Jul 2021

4. Elrod JK, Fortenberry JL (2017) The hub-and-spoke organization design: an avenue for serving patients well. BMC Health Serv Res 17:457

5. COVID Surg Collaborative (2020) Elective surgery cancellations due to the COVID-19 pandemic: global predictive modelling to inform surgical recovery plans. Br J Surg 107:1440-1449

6. COVID Live Update: $189,954,148$ Cases and 4,086,616 deaths from the coronavirus-Worldometer. Available from: https:// www.worldometers.info/coronavirus/. Accessed 16 Jul 2021

7. Pergolini I, Demir IE, Stöss C et al (2021) Effects of COVID-19 pandemic on the treatment of pancreatic cancer: a perspective from Central Europe. Dig Surg 38:158-165

8. Oba A, Stoop TF, Löhr M et al (2020) Global survey on pancreatic surgery during the COVID-19 pandemic. Ann Surg 272:e87-e93

9. Balakrishnan A, Lesurtel M, Siriwardena AK et al (2020) Delivery of hepato-pancreato-biliary surgery during the COVID-19 pandemic: an European-African Hepato-Pancreato-Biliary Association (E-AHPBA) cross-sectional survey. HPB 22:1128-1134

10. Marchegiani G, Paiella S, Malleo G et al (2020) Love (pancreatic surgery) in the time of cholera (COVID-19). Dig Surg 37:524-526

11. Hata T, Motoi F, Ishida M et al (2016) Effect of hospital volume on surgical outcomes after pancreaticoduodenectomy: a systematic review and meta-analysis. Ann Surg 263:664-672

12. Latenstein AEJ, Mackay TM, van der Geest LGM et al (2021) Effect of centralization and regionalization of pancreatic surgery on resection rates and survival. Br J Surg 108(7):826-833

13. Balzano G, Guarneri G, Pecorelli N et al (2020) Modelling centralization of pancreatic surgery in a nationwide analysis. Br J Surg 107:1510-1519

14. Balzano G, Zerbi A, Capretti G et al (2008) Effect of hospital volume on outcome of pancreaticoduodenectomy in Italy. Br J Surg 95:357-362

15. Dindo D, Demartines N, Clavien P-A (2004) Classification of surgical complications: a new proposal with evaluation in a cohort of 6336 patients and results of a survey. Ann Surg 240:205-213

16. COVID Surg Collaborative (2020) Global guidance for surgical care during the COVID-19 pandemic. Br J Surg 107:1097-1103

17. Lazzati A, Raphael Rousseau M, Bartier S et al (2021) Impact of COVID-19 on surgical emergencies: nationwide analysis. BJS Open 5:zrab039

18. COVID Surg Collaborative (2020) Mortality and pulmonary complications in patients undergoing surgery with perioperative SARS-CoV-2 infection: an international cohort study. Lancet Lond Engl 396:27-38

19. Torzilli G, Viganò L, Galvanin J et al (2020) A snapshot of elective oncological surgery in Italy during COVID-19 emergency: pearls, pitfalls, and perspectives. Ann Surg 272:e112-e117

20. Yu J, Blackford AL, dal Molin M et al (2015) Time to progression of pancreatic ductal adenocarcinoma from low-to-high tumour stages. Gut 64:1783-1789 
21. Marchegiani G, Andrianello S, Perri G et al (2018) Does the surgical waiting list affect pathological and survival outcome in resectable pancreatic ductal adenocarcinoma? HPB 20:411-417

22. Müller PC, Hodson J, Kuemmerli C et al (2020) Effect of time to surgery in resectable pancreatic cancer: a systematic review and meta-analysis. Langenbecks Arch Surg 405:293-302

23. Moslim MA, Hall MJ, Meyer JE et al (2021) Pancreatic cancer in the era of COVID-19 pandemic: which one is the lesser of two evils? World J Clin Oncol 12:54-60

24. Tzeng CWD, Teshome M, Katz MHG et al (2020) Cancer surgery scheduling during and after the COVID-19 first wave: The MD Anderson Cancer Center experience. Ann Surg 272:106
25. Salvia R, Andrianello S, Ciprani D et al (2021) Pancreatic surgery is a safe teaching model for tutoring residents in the setting of a high-volume academic hospital: a retrospective analysis of surgical and pathological outcomes. HPB 23:520-527

Publisher's Note Springer Nature remains neutral with regard to jurisdictional claims in published maps and institutional affiliations. 